University of Nebraska - Lincoln

DigitalCommons@University of Nebraska - Lincoln

Norman R. Simon Papers

Research Papers in Physics and Astronomy

3-15-1985

\title{
The Unusual Pulsating Variable XZ Ceti
}

Terry J. Teays

University of Nebraska-Lincoln, tteays1@jhu.edu

Norman R. Simon

University of Nebraska-Lincoln, nsimon@unl.edu

Follow this and additional works at: https://digitalcommons.unl.edu/physicssimon

Teays, Terry J. and Simon, Norman R., "The Unusual Pulsating Variable XZ Ceti" (1985). Norman R. Simon Papers. 31.

https://digitalcommons.unl.edu/physicssimon/31

This Article is brought to you for free and open access by the Research Papers in Physics and Astronomy at DigitalCommons@University of Nebraska - Lincoln. It has been accepted for inclusion in Norman R. Simon Papers by an authorized administrator of DigitalCommons@University of Nebraska - Lincoln. 


\title{
THE UNUSUAL PULSATING VARIABLE XZ CETI
}

\author{
TERRY J. TEAYS ${ }^{1}$ AND NORMAN R. SimON \\ Department of Physics and Astronomy, University of Nebraska-Lincoln \\ Received 1984 July 2 ; accepted 1984 September 25
}

\begin{abstract}
The peculiar pulsating variable XZ Ceti has a period of 0.8231 day, suggesting that it is a Bailey type $a b$ RR Lyrae star, but it has a light curve shape and amplitude which is like that of the Bailey type $c$ variables. Fourier decomposition analysis verifies that the parameters of its light curve match those of the type $c$ variables, except for its unacceptably long period. We have obtained a new photometric light curve which verifies this long period. We have also obtained energy distributions from spectrum scans, which are used to derive a temperature and approximate surface gravity of this star. These results are used to construct pulsation models so as to attempt to identify the nature of $\mathrm{XZ}$ Ceti. The models suggest the possibility that $\mathrm{XZ}$ Ceti is an anomalous Cepheid or, perhaps, an overtone BL Her star.
\end{abstract}

Subject headings: stars: individual — stars: pulsation - stars: RR Lyrae

\section{INTRODUCTION}

$\mathrm{XZ}$ Ceti is a small-amplitude pulsator originally identified as an RR Lyrae star (Kukarkin et al. 1969). A detailed light curve obtained by Dean et al. (1977) indicated a period of 0.8231 day and a $V$ amplitude of 0.49 mag. While the period of this star suggests a fundamental-mode pulsator (Bailey type $a b$ ), its low amplitude and sinusoidal light curve are reminiscent of pulsation in the first overtone (Bailey type $c$ ). More precisely, when the light curve of XZ Ceti was Fourier-decomposed and the coefficients plotted on the Fourier coefficient diagrams of Simon and Teays (1982), the star was found to fall far from the region occupied by the type $a b$ pulsators.

If XZ Ceti has a normal Population II mass and pulsates in an overtone, then it is a BL Her star, perhaps the first example of overtone pulsation in this class of stars. Alternatively, Simon and Teays (1982) suggested the possibility that XZ Ceti might be a fundamental-mode pulsator whose light curve has been strongly modified by a near resonance in its period spectrum. This idea was based upon an analogy with the Hertzsprung progression among Population I Cepheids (Simon and Lee 1981).

In addition to the above explanations, a third possibility merits consideration, viz., that XZ Ceti belongs to the class of "anomalous Cepheids" (Zinn 1980). The anomalous Cepheids, found in dwarf spheroidal galaxies and in the SMC, do not obey the period-luminosity relation of either the classical or Population II Cepheids. These stars are believed to have gained mass from a binary companion, because their high luminosities indicate masses 2-3 times larger than those of Population II Cepheids (Renzini, Mengel, and Sweigart 1977). The only known example of an anomalous Cepheid in the Galaxy is the star V19 in the globular cluster NGC 5466 (Zinn and King 1982). V19 has a period of 0.821 day and a sinusoidal light curve, with a $V$ amplitude of $0.64 \mathrm{mag}$. We have performed a Fourier decomposition analysis of the light curve of V19, and find the following values for the coefficients:

$$
\phi_{21}(\mathrm{~V} 19)=4.55, \quad R_{21}(\mathrm{~V} 19)=7.16 \times 10^{-2} .
$$

\footnotetext{
${ }^{1}$ Guest Observer, Cerro Tololo Inter-American Observatory, National Optical Astronomy Observatories, which is operated by the Association of Universities for Research in Astronomy, Inc., under contract with the National Science Foundation.
}

Plotting these values on the Fourier diagrams of Simon and Teays (1982) one finds that V19 falls between XZ Ceti and the RR Lyrae field stars on the $\phi_{21}$ plot and is alone with XZ Ceti in the lower right corner of the $R_{21}$ plot. Thus the qualitative similarities between the two light curves are verified reasonably well by the Fourier analysis. The correspondence is close enough that one must consider the possibility that XZ Ceti, like V19, is an anomalous Cepheid.

In the present work we shall describe new observations of $\mathrm{XZ}$ Ceti designed to verify the published period and to obtain estimates of the temperature and surface gravity of this object. With these estimates in hand we shall build pulsation models of XZ Ceti and use them to try to determine the nature of this interesting star.

\section{PHOTOMETRY}

The light curve of Dean et al. (1977) was obtained by combining observations from many different nights. It is a wellknown fact that many RR Lyrae star light curves do not repeat from one cycle to the next, and so one must be cautious in accepting such a curve for detailed analysis. It was therefore necessary to obtain photometry on a number of successive nights to verify the period and specific shape of the light curve. Broad-band (Johnson) $B$ and $V$ observations were obtained with the $91 \mathrm{~cm}$ telescope of Cerro Tololo Inter-American Observatory. Unusually poor weather for November at Cerro Tololo prevented us from acquiring the complete phase coverage which is necessary to perform Fourier analysis of the type used in Simon and Teays (1982), but some data were obtained on five of the seven allotted nights.

Differential photometry was obtained, using the star SAO 148173 as a comparison and SAO 148183 as a check star. Since SAO 148173 is not a well-observed star, it was necessary to establish its constancy; the differences between SAO 148173 and SAO 148183 during the course of the observations are shown in Figure 1, and verify its nonvariability. The differential photometry was not corrected for air mass difference between the comparison and variable, due to their proximity in the sky. XZ Ceti (SAO 148149) is located at $\alpha=1^{\mathrm{h}} 57^{\mathrm{m}} 52^{\mathrm{s}} .6, \delta=$ $-16^{\circ} 35^{\prime} 15^{\prime \prime}$ (1950); while SAO 148173 is located at $\alpha=$ $2^{\mathrm{h}} 1^{\mathrm{m}} 2^{\mathrm{s}} 0, \delta=-17^{\circ} 16^{\prime} 23^{\prime \prime}$. Table 1 gives the results of the photometry. The first column gives the heliocentric Julian date, the 


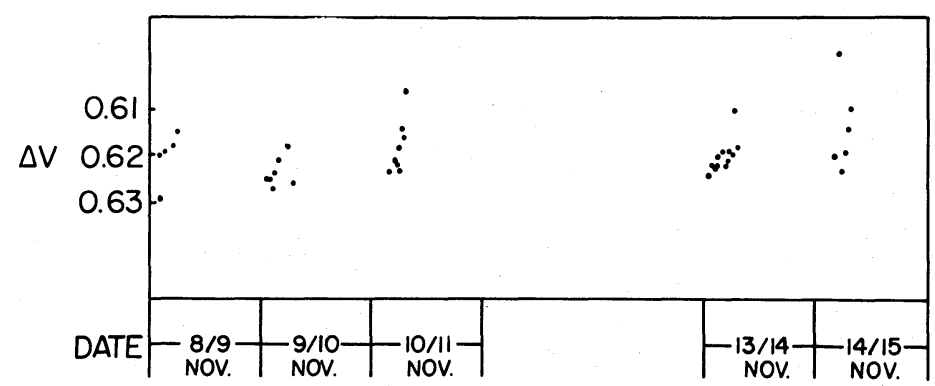

Fig. 1.-Differential magnitude between comparison star (SAO 148173) and check star (SAO 148183). No extinction or color corrections have been applied.

second column is the phase (with respect to maximum light), the third column gives the differential color index, and the fourth column lists the magnitude difference between variable and comparison. The differential magnitude is given rather than the $V$ magnitude, due to its higher accuracy, but a standard star solution yielded a $V$ magnitude for SAO 148173 of 8.260 , from which the $V$ magnitude of XZ Ceti may be inferred. The comparison star was chosen to have a color as close to the variable's as possible, so the color correction terms were quite small ( $\leq 0.002 \mathrm{mag})$. The $V$ light curve of $\mathrm{XZ}$ Ceti is shown in Figure 2.

\section{ENERGY DISTRIBUTIONS}

In order to estimate the temperature of $\mathrm{XZ}$ Ceti, energy distributions were obtained using the Harvard two-channel scanner, on the Cerro Tololo $1.5 \mathrm{~m}$ telescope. Observations were made between 3390 and $8090 \AA$ at the wavelengths used by Oke (1964). Unfortunately, weather and instrument prob-

TABLE 1

Differential Photometry (XZ CeTI-SAO 148173)

\begin{tabular}{|c|c|c|c|c|c|c|c|}
\hline $\begin{array}{c}\text { HJD } \\
2,445,200+\end{array}$ & $\phi$ & $\Delta(B-V)$ & $\Delta V$ & $\begin{array}{c}\text { HJD } \\
2,445,200+\end{array}$ & $\phi$ & $\Delta(B-V)$ & $\Delta V$ \\
\hline $82.5415 \ldots$ & 0.341 & 0.103 & 1.390 & 87.5724 . & 0.453 & 0.147 & 1.492 \\
\hline $82.5537 \ldots$ & 0.356 & 0.123 & 1.392 & 87.5767. & 0.458 & 0.152 & 1.498 \\
\hline $82.6110 \ldots$ & 0.425 & 0.140 & 1.480 & $87.5874 \ldots$ & 0.471 & 0.155 & 1.512 \\
\hline $82.6591 \ldots$ & 0.484 & 0.159 & 1.523 & $87.5914 \ldots$ & 0.476 & 0.153 & 1.517 \\
\hline $82.6653 \ldots$ & 0.491 & 0.161 & 1.527 & $87.6143 \ldots$ & 0.504 & 0.164 & 1.539 \\
\hline $82.7062 \ldots$ & 0.541 & 0.165 & 1.558 & $87.6161 \ldots$ & 0.506 & 0.165 & 1.540 \\
\hline $82.7390 \ldots$ & 0.581 & 0.161 & 1.578 & $87.6182 \ldots$ & 0.509 & 0.165 & 1.542 \\
\hline $82.7415 \ldots$ & 0.584 & 0.164 & 1.580 & $87.6200 \ldots$ & 0.511 & 0.165 & 1.542 \\
\hline $82.7446 \ldots$ & 0.588 & 0.166 & 1.586 & $87.6215 \ldots$ & 0.513 & 0.165 & 1.548 \\
\hline $82.7485 \ldots$ & 0.593 & 0.169 & 1.592 & $87.6432 \ldots$ & 0.539 & 0.167 & 1.556 \\
\hline $82.7877 \ldots$ & 0.640 & 0.164 & 1.586 & $87.6471 \ldots$ & 0.544 & 0.170 & 1.561 \\
\hline $82.8208 \ldots \ldots \ldots$ & 0.680 & 0.164 & 1.574 & $87.6579 \ldots$ & 0.557 & 0.172 & 1.567 \\
\hline $83.5116 \ldots$ & 0.520 & 0.157 & 1.554 & $87.6625 \ldots$ & 0.563 & 0.174 & 1.565 \\
\hline $83.5190 \ldots$ & 0.529 & 0.164 & 1.552 & $87.6723 \ldots$ & 0.575 & 0.165 & 1.576 \\
\hline $83.5252 \ldots$ & 0.536 & 0.162 & 1.560 & $87.6781 \ldots \ldots \ldots$ & 0.582 & 0.167 & 1.578 \\
\hline $83.5565 \ldots$ & 0.574 & 0.170 & 1.568 & $87.6988 \ldots \ldots \ldots$ & 0.607 & 0.171 & 1.585 \\
\hline $83.5616 \ldots$ & 0.580 & 0.167 & 1.575 & $87.7036 \ldots$ & 0.613 & 0.166 & 1.590 \\
\hline $83.5895 \ldots$ & 0.614 & 0.167 & 1.585 & $87.7069 \ldots$ & 0.617 & 0.165 & 1.587 \\
\hline $83.5951 \ldots \ldots \ldots$ & 0.621 & 0.171 & 1.583 & $87.7156 \ldots$ & 0.627 & 0.156 & 1.595 \\
\hline $83.6296 \ldots \ldots \ldots$ & 0.663 & 0.177 & 1.574 & $87.7195 \ldots$ & 0.632 & 0.160 & 1.593 \\
\hline $83.6630 \ldots \ldots \ldots$ & 0.704 & 0.157 & 1.562 & $87.7219 \ldots$ & 0.635 & 0.158 & 1.592 \\
\hline $83.6643 \ldots \ldots \ldots$ & 0.705 & 0.156 & 1.558 & $87.7480 \ldots \ldots \ldots$ & 0.667 & 0.156 & 1.591 \\
\hline $83.6922 \ldots \ldots \ldots$ & 0.739 & 0.141 & 1.510 & $87.7518 \ldots$ & 0.671 & 0.158 & 1.584 \\
\hline $83.7645 \ldots$ & 0.827 & 0.086 & 1.307 & $87.7701 \ldots$ & 0.693 & 0.151 & 1.582 \\
\hline $83.7712 \ldots$ & 0.835 & 0.066 & 1.308 & $87.7746 \ldots$ & 0.699 & 0.157 & 1.571 \\
\hline $83.8042 \ldots$ & 0.875 & 0.053 & 1.195 & $87.7856 \ldots \ldots \ldots$ & 0.712 & 0.155 & 1.554 \\
\hline 83.8087. & 0.881 & 0.046 & 1.178 & $87.7911 \ldots \ldots \ldots$ & 0.719 & 0.148 & 1.553 \\
\hline $84.6759 \ldots \ldots \ldots$ & 0.934 & 0.018 & 1.148 & $87.7967 \ldots \ldots \ldots$ & 0.726 & 0.146 & 1.537 \\
\hline $84.6929 \ldots \ldots \ldots$ & 0.955 & 0.023 & 1.134 & $87.8168 \ldots \ldots \ldots$ & 0.750 & 0.143 & 1.523 \\
\hline $84.7011 \ldots \ldots \ldots$ & 0.965 & 0.021 & 1.130 & $88.6584 \ldots \ldots \ldots$ & 0.773 & 0.121 & 1.435 \\
\hline $84.7159 \ldots \ldots \ldots$ & 0.983 & 0.021 & 1.125 & $88.6673 \ldots$ & 0.783 & 0.096 & 1.427 \\
\hline $84.7301 \ldots \ldots \ldots$ & 0.000 & 0.019 & 1.126 & $88.6717 \ldots \ldots \ldots$ & 0.789 & 0.096 & 1.407 \\
\hline $84.7430 \ldots \ldots \ldots$ & 0.016 & 0.026 & 1.126 & $88.6842 \ldots$ & 0.804 & 0.097 & 1.373 \\
\hline $84.7579 \ldots \ldots \ldots$ & 0.034 & 0.025 & 1.136 & $88.6890 \ldots$ & 0.810 & 0.093 & 1.359 \\
\hline $84.7719 \ldots \ldots \ldots$ & 0.051 & 0.027 & 1.132 & $88.7166 \ldots$ & 0.843 & 0.084 & 1.294 \\
\hline $84.8032 \ldots \ldots \ldots$ & 0.089 & 0.038 & 1.149 & $88.7230 \ldots \ldots \ldots$ & 0.851 & 0.072 & 1.278 \\
\hline $87.5152 \ldots \ldots \ldots$ & 0.384 & 0.136 & 1.419 & $88.7562 \ldots \ldots \ldots$ & 0.891 & 0.040 & 1.193 \\
\hline $87.5205 \ldots \ldots \ldots$ & 0.390 & 0.136 & 1.432 & $88.7618 \ldots \ldots \ldots$ & 0.898 & 0.048 & 1.179 \\
\hline $87.5482 \ldots \ldots \ldots$ & 0.424 & 0.143 & 1.465 & $88.7861 \ldots \ldots \ldots$ & 0.928 & 0.029 & 1.147 \\
\hline $87.5544 \ldots \ldots \ldots$ & 0.431 & 0.144 & 1.473 & $88.8074 \ldots \ldots \ldots$ & 0.954 & 0.031 & 1.132 \\
\hline
\end{tabular}




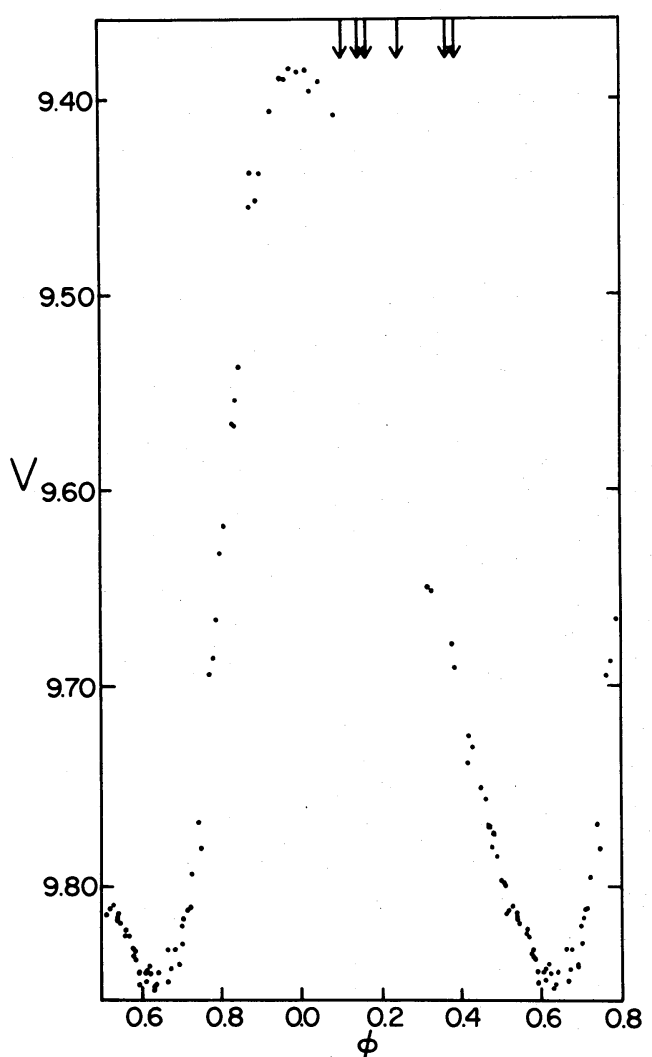

FIG. 2.- $V$ light curve of $\mathrm{XZ}$ Ceti. Arrows mark the position in phase at which the energy distributions were obtained. lems on both of the nights prevented us from obtaining scans throughout the pulsation cycle. Additional problems which resulted from the abbreviated observing time both nights were that only a limited number of standard stars could be observed, and, especially, that there was an insufficient range in air mass for standard stars to sufficiently determine extinction. The scans were, therefore, corrected for extinction by using the mean extinction coefficients for 1980 July/August at each wavelength, as given by Gutierrez-Moreno, Moreno, and Cortes (1982) for Cerro Tololo.

A single scan consisted of measurements at 20 wavelengths, starting with the shortest, and then repeating the sequence in reverse. This standard procedure provides the best average air mass for the scan. A complete scan took approximately 6 minutes. To determine each energy distribution, several consecutive scans were added together. The final result was six energy distributions. The instrumental fluxes were converted to standard magnitudes by comparing them with the values for these standards which are cited in Breger's (1978) catalog, which is based on the Hayes and Latham (1975) calibration of Vega.

Lacking any specific information about the color excess of this star, we made no correction to the data for interstellar reddening. This is probably a reasonable assumption given that the galactic latitude of XZ Ceti is approximately $-71^{\circ}$.

Table 2 gives the logarithm of the fluxes at each wavelength for the six composite scans. These data have been normalized to 0 at $5000 \AA$. The phase at which the scans were taken is indicated by arrows in Figure 2. Table 3 gives some details of the composite scans: the number of scans that were added together to form the composite, the mean sidereal time of the observations, the phase of this observation, the inferred $B-V$ color index, the temperature derived for it, and the logarithm

TABLE 2

$\log f_{\lambda}$, AT EACH WAVELENGTH, FOR THE SCANS ${ }^{a}$

\begin{tabular}{|c|c|c|c|c|c|c|}
\hline \multirow[b]{2}{*}{$\begin{array}{l}\text { WAVELENGTH } \\
(\AA)\end{array}$} & \multicolumn{6}{|c|}{$\begin{array}{l}\text { SCAN NUMBER } \\
\text { DATE } \\
\text { RuN NUMBERS }\end{array}$} \\
\hline & $\begin{array}{c}1 \\
\text { Nov } 7 / 8 \\
8-11\end{array}$ & $\begin{array}{c}2 \\
\text { Nov } 6 / 7 \\
21-29\end{array}$ & $\begin{array}{c}3 \\
\text { Nov } 7 / 8 \\
18-22\end{array}$ & $\begin{array}{c}4 \\
\text { Nov } 7 / 8 \\
30-36\end{array}$ & $\begin{array}{c}5 \\
\text { Nov } 7 / 8 \\
45-48\end{array}$ & $\begin{array}{c}6 \\
\text { Nov } 7 / 8 \\
49-52\end{array}$ \\
\hline 3390 . & -0.322 & -0.303 & -0.317 & -0.312 & -0.297 & -0.290 \\
\hline $3448 \ldots$ & -0.309 & -0.300 & -0.305 & -0.293 & -0.274 & -0.277 \\
\hline $3509 \ldots$ & -0.310 & -0.295 & -0.301 & -0.290 & -0.274 & -0.276 \\
\hline $3570 \ldots \ldots \ldots$ & -0.300 & -0.286 & -0.286 & -0.281 & -0.266 & -0.263 \\
\hline $3636 \ldots \ldots \ldots$ & -0.296 & -0.280 & -0.282 & -0.270 & -0.267 & -0.255 \\
\hline $4036 \ldots \ldots \ldots$ & +0.149 & +0.167 & +0.147 & +0.140 & +0.119 & +0.115 \\
\hline $4167 \ldots \ldots \ldots$ & +0.131 & +0.148 & +0.130 & +0.118 & +0.099 & +0.098 \\
\hline $4255 \ldots \ldots \ldots$ & +0.111 & +0.128 & +0.116 & +0.106 & +0.088 & +0.086 \\
\hline $4464 \ldots \ldots \ldots$ & +0.092 & +0.105 & +0.088 & +0.084 & +0.075 & +0.075 \\
\hline $4566 \ldots \ldots \ldots$ & +0.064 & +0.064 & +0.071 & +0.061 & +0.051 & +0.051 \\
\hline $4780 \ldots \ldots \ldots$ & +0.035 & +0.046 & +0.039 & +0.033 & +0.027 & +0.032 \\
\hline $5000 \ldots \ldots \ldots$ & 0.000 & 0.000 & 0.000 & 0.000 & 0.000 & 0.000 \\
\hline $5264 \ldots \ldots \ldots$ & -0.030 & -0.018 & -0.027 & -0.032 & -0.025 & -0.027 \\
\hline $5556 \ldots \ldots \ldots$ & -0.072 & -0.064 & -0.070 & -0.067 & -0.061 & -0.052 \\
\hline $5840 \ldots$ & -0.110 & -0.103 & -0.109 & -0.091 & -0.090 & -0.087 \\
\hline $6050 \ldots \ldots \ldots$ & -0.137 & -0.136 & -0.135 & -0.128 & -0.118 & -0.119 \\
\hline $7100 \ldots \ldots \ldots$ & -0.257 & -0.262 & -0.248 & -0.248 & -0.224 & -0.211 \\
\hline $7530 \ldots \ldots \ldots$ & -0.312 & $\ldots$ & -0.303 & -0.295 & -0.271 & -0.256 \\
\hline $7550 \ldots \ldots \ldots$ & -0.329 & -0.321 & -0.318 & -0.310 & -0.279 & -0.274 \\
\hline $8090 \ldots \ldots \ldots$ & -0.376 & -0.386 & -0.367 & -0.351 & -0.314 & -0.304 \\
\hline
\end{tabular}

a The scans have been normalized to 0 at $5000 \AA$. 
TABLE 3

SCAN RESULTS

\begin{tabular}{lcccccc}
\hline \hline $\begin{array}{c}\text { Scan } \\
\text { Number }\end{array}$ & $\begin{array}{c}\text { Number of } \\
\text { Individual } \\
\text { Scans }\end{array}$ & $\begin{array}{c}\text { Mean } \\
\text { UT of } \\
\text { Observations }\end{array}$ & $\phi$ & $\begin{array}{c}\text { Approximate } \\
(B-V)\end{array}$ & $T_{\text {eff }}$ & $\log g$ \\
\hline $1 \ldots \ldots \ldots$ & 4 & $00: 49: 37$ & 0.112 & 0.290 & 6770 & 2.25 \\
$2 \ldots \ldots \ldots \ldots$ & 9 & $05: 49: 44$ & 0.150 & 0.301 & 6950 & 2.40 \\
$3 \ldots \ldots \ldots$ & 5 & $01: 57: 30$ & 0.169 & 0.311 & 6650 & 2.00 \\
$4 \ldots \ldots \ldots$ & 7 & $03: 31: 14$ & 0.248 & 0.338 & 6530 & 2.15 \\
$5 \ldots \ldots \ldots$ & 4 & $05: 51: 04$ & 0.366 & 0.371 & 6425 & 2.00 \\
$6 \ldots \ldots \ldots$ & 4 & $06: 20: 07$ & 0.391 & 0.380 & 6325 & 2.00 \\
\hline
\end{tabular}

of the surface gravity. All of the scans were obtained on 1982 November $7 / 8$ except for scan 2, which was obtained on November 6/7.

\section{TEMPERATURE}

In order to determine the temperature, the scans were fitted to the theoretical model atmospheres of Kurucz (1979). Consideration of the possible nature of $\mathrm{XZ}$ Ceti suggests a Population II abundance for this star, and this notion is strengthened by its fairly large distance above the galactic plane. The energy distributions were therefore compared to the Kurucz models with $\log Z=-2$. Although we lack any specific information regarding the abundances of XZ Ceti, a comparison of model atmospheres with the same temperature but different abundances indicates the slope of the continuum longward of the Balmer jump is not strongly dependent upon composition.

The temperatures that were derived from these fits, interpolated by eye, are listed in Table 3 . The temperatures listed represent an average between the eye estimates of one of us (T. J. T.) and those of Schmidt (1984), which were in close agreement. These six temperatures are inadequate to determine an average temperature for XZ Ceti due to their limited range of phase. To resolve this problem, the $B-V$ color curve was constructed from the photometry and a relation was determined between the temperatures derived from the scans and those derived from the colors. Unfortunately, as is obvious from Figure 2, the scans from which the temperatures were obtained happened to occur, for the most part, during the phases for which it was not possible to secure photometry. The best that could be done was to sketch in the remainder of the curve, under the assumption that it varied smoothly across this gap in phase, a presumption which is supported by the descending branch of the light curves obtained by Dean et al. (1977). A linear regression analysis was done, and the relation between the temperature and the respective color indices for these six points was computed. The result was:

$$
B-V=-1.492 \times 10^{-4} T+1.318 .
$$

TABLE 4

MODEL PARAMETERs ${ }^{\mathrm{a}}$

\begin{tabular}{cccccc}
\hline \hline $\begin{array}{c}\text { Model } \\
\text { Number }\end{array}$ & $M / M_{\odot}$ & $L / L_{\odot}$ & $M_{B}$ & $\log g$ & $\begin{array}{c}\text { Model } \\
\text { Type }\end{array}$ \\
\hline $1 \ldots \ldots \ldots$ & 0.65 & 72.8 & +0.57 & 2.6 & RR-F \\
$2 \ldots \ldots \ldots$ & 0.23 & 33.0 & +1.42 & 2.5 & RR-F \\
$3 \ldots \ldots \ldots$ & 2.50 & 211 & -0.59 & 2.7 & AC-F \\
$4 \ldots \ldots \ldots$ & 0.60 & 101 & +0.21 & 2.4 & BL-O \\
$5 \ldots \ldots \ldots$ & 1.50 & 205 & -0.56 & 2.5 & AC-O \\
\hline
\end{tabular}

${ }^{\mathrm{a}} T_{\text {eff }}=6500 \mathrm{~K}, X=0.70, Z=0.001$.

The average $\langle B-V\rangle$ was then calculated by averaging points selected at each 0.1 in phase. ${ }^{2}$ The average color index yielded by this procedure was 0.343 . This was then converted to an average temperature via equation (1). The final result is that the average temperature for XZ Ceti is $6540 \mathrm{~K}$. It is this temperature that we will use in our discussion below.

The models and the scans were also compared with respect to the size of the Balmer jump, in order to deduce an approximate surface gravity. The comparison of observations of a pulsating stellar atmosphere to models of a static atmosphere is a questionable procedure, but it should serve as a rough estimate. All the scans occur during the descending branch of the light curve. These estimates of surface gravity are listed in Table 3 and are discussed in the theoretical analysis below. The gravities obtained are unexpectedly low, falling close to log $g=2.0$. Note that Zinn and King (1982) found essentially the same values for the anomalous Cepheid V19, using the same model atmospheres as we have.

\section{THE MODELS}

Linear nonadiabatic (LNA) pulsation models were integrated using the conventional Lagrangean code described by Aikawa and Simon (1983). The models all had Population II composition $(X=0.70, Z=0.001)$ and an effective temperature, $T_{\text {eff }}=6500 \mathrm{~K}$, corresponding to the temperature obtained from the observations of XZ Ceti described in earlier sections. Table 4 displays the model parameters: mass, luminosity, estimated blue absolute magnitude, and log of surface gravity. The last column gives a brief characterization of the model: $\mathrm{RR}=\mathrm{RR}$ Lyrae, $\mathrm{BL}=\mathrm{BL}$ Her, $\mathrm{AC}=$ anomalous Cepheid; $\mathrm{F}=$ fundamental, $\mathrm{O}=$ first overtone.

Table 5 presents the pulsation model results: fundamental period and growth rate (notation as in Aikawa and Simon 1983), first-overtone period and growth rate, ratio of second

\footnotetext{
2 This average was used instead of the normally preferable $\langle B\rangle-\langle V\rangle$ because of the necessity of bridging the phase gap by eye. Because the color curve has a lower amplitude than either $V$ or $B$, there is a smaller error when the curve is sketched in by hand.
}

TABLE 5

Pulsation Results ${ }^{\mathrm{a}}$

\begin{tabular}{lcccrrrrr}
\hline $\begin{array}{c}\text { Model } \\
\text { Number }\end{array}$ & $P_{0}$ & $\eta_{0}$ & $P_{1}$ & \multicolumn{1}{c}{$\eta_{1}$} & \multicolumn{1}{c}{$P_{2} / P_{0}$} & \multicolumn{1}{c}{$\eta_{2}$} & \multicolumn{1}{c}{$P_{3} / P_{0}$} & $\eta_{3}$ \\
\hline $1 \ldots \ldots \ldots$ & $\mathbf{0 . 8 2}$ & $3.8(-2)$ & 0.60 & $7.3(-2)$ & 0.57 & $-5.2(-2)$ & 0.46 & $-2.1(-1)$ \\
$2 \ldots \ldots \ldots$ & $\mathbf{0 . 8 6}$ & $1.7(-1)$ & 0.59 & $-2.8(-2)$ & 0.50 & $-4.1(-1)$ & 0.39 & $-4.3(-1)$ \\
$3 \ldots \ldots \ldots$ & $\mathbf{0 . 8 2}$ & $1.8(-3)$ & 0.64 & $1.0(-2)$ & 0.63 & $2.7(-2)$ & 0.53 & $1.4(-2)$ \\
$4 \ldots \ldots \ldots$ & 1.14 & $5.9(-2)$ & $\mathbf{0 . 8 2}$ & $5.7(-2)$ & 0.54 & $-1.9(-1)$ & 0.43 & $-3.9(-1)$ \\
$5 \ldots \ldots \ldots$ & 1.11 & $1.0(-2)$ & $\mathbf{0 . 8 3}$ & $4.6(-2)$ & 0.60 & $1.4(-2)$ & 0.49 & $-8.6(-2)$ \\
\hline
\end{tabular}

a Period $P$ in days; growth rate $\eta$ in percent per period. 
overtone to fundamental period, second-overtone growth rate, ratio of third overtone to fundamental period, and thirdovertone growth rate. For each model the period of the mode meant to represent XZ Ceti is entered in boldface. Since the models are preliminary no attempt was made to match the calculated period exactly to that of the star.

Model 1 falls within the range of masses and luminosities calculated by Cox (1980) but is somewhat overluminous compared with the canonical value for RR Lyrae stars (Stothers 1983). The choice of luminosity was necessary to match the period of $\mathrm{XZ}$ Ceti. However, this model has period ratios $\left(P_{2} / P_{0}=0.57, P_{3} / P_{0}=0.46\right)$ which are far from resonance. To construct a resonant model one can impose the resonance condition (say, $P_{2} / P_{0}=0.5$ ), in which case the mass and luminosity will be determined for any given temperature. With $T=6500 \mathrm{~K}$, corresponding to XZ Ceti, we obtain model 2 . This model is indeed resonant, but its mass is $0.23 M_{\odot}$, far too low for a normal RR Lyrae star. While a resonant model with acceptable mass could be constructed for $P_{3} / P_{0}=0.5$, there is another line of evidence which goes toward ruling out any fundamental mode (F-mode) RR Lyrae explanation for XZ Ceti.

Petersen (1984) has plotted XZ Ceti on Fourier decomposition diagrams (Simon and Teays 1982) with stars of similar period from the globular cluster $\omega$ Cen. While the latter stars (presumably F-mode pulsators) form an orderly sequence in the Fourier quantities $\phi_{21}$ and $R_{21}, \mathrm{XZ}$ Ceti lies far from these points, falling well above on the $\phi_{21}$ diagram and well below on the $R_{21}$ diagram. On the other hand, Petersen shows that, apart from XZ Ceti, the other field stars in the sample of Simon and Teays (1982) have Fourier coefficients which fit well with the stars of $\omega$ Cen. Thus there are two different arguments which seem to converge toward the conclusion that XZ Ceti is not a F-mode RR Lyrae star.

Model 4 has standard parameters for a BL Her star (see, e.g., Carson, Stothers, and Vemury 1981). While its first overtone period corresponds to that of XZ Ceti, it can be seen from Table 5 that the linear growth rate $\eta_{1}$ of this mode is slightly less than the growth rate $\eta_{0}$ of the fundamental. Although it is not generally possible to predict limiting amplitude behavior from linear models, it has nonetheless been the experience of a number of investigators that when both modes are linearly unstable, the fundamental limit cycle will be selected if its linear growth rate approaches or exceeds that of the overtone (King et al. 1973; Stellingwerf 1975). We note that model 4 lies in the region of the H-R diagram in which the first-overtone blue edge has already crossed redward of the fundamental blue edge (Cox 1980). Indeed, hydrodynamic calculations indicate that model 4 ought to fall in the F-mode-only domain in both the Stellingwerf (1975) and Spangenberg (1975) stability diagrams (Cox 1980). Thus we can tentatively explain the observational result that all BL Her stars seem to be pulsating in the fundamental mode: these stars lie in a region of the H-R diagram characterized by F-mode-only pulsation.

Models 3 and 5 are anomalous Cepheid models with parameters similar to those given by Zinn and King (1982) for V19. In model 3 the fundamental period matches that of $\mathrm{XZ}$ Ceti, but the first, second, and third overtones are also unstable, with linear growth rates exceeding that of the fundamental by factors of 6,15 , and 8 respectively. In addition, the mass of this model is probably unacceptably large to have resulted from binary mass exchange in a Population II system (Renzini, Mengel, and Sweigart 1977). Some further arguments against this model will be presented below.
Model 5 represents an anomalous Cepheid pulsating in the first overtone. Its mass is equal to the "typical" mass given by Renzini, Mengel, and Sweigart (1977) for anomalous Cepheids, assuming the canonical value $M=0.65 M_{\odot}$ for $\mathrm{RR}$ Lyrae stars. While this model is linearly unstable in the first three normal modes, its first overtone growth rate exceeds that of the fundamental by a factor $>4$, and that of the second overtone by a factor $>3$. Although it needs to be emphasized again that we cannot translate linear growth rates directly into limit cycles, this result certainly seems favorable for the first overtone.

The surface gravity of model 5 is $\log g=2.5$. The handful of gravity measurements for XZ Ceti seem to indicate that this value is too high, as are the gravities for the other models in Table 4. However, as pointed out earlier, the gravity of Zinn and King's (1982) preferred model for V19 is also high with respect to actual measurements of $\log g$. These results could indicate that the model parameters are incorrect, but more likely they reflect underestimation of the gravities of the respective stars. Accurate determination of the static gravity of a pulsating star constitutes a long-standing problem in variable star research (see, e.g., the discussion and references in Simon 1979). For the case of XZ Ceti the discrepancy in $\log g$ is worst for two of the F-mode models, viz., models 1 and 3.

\section{DISCUSSION}

Five LNA pulsation models have been constructed for the small-amplitude variable $\mathrm{XZ}$ Ceti. Of these, three have an F-mode period corresponding to that of the star (models 1, 2, and 3), while the remaining two are first-overtone models. In the previous section a number of arguments have been advanced against the F-mode interpretation. Models 1 and 3 do not have appropriate period ratios to provide a resonance explanation for the low-amplitude, sinusoidal light curve of $\mathrm{XZ}$ Ceti. These two models also display slightly larger discrepancies with the estimated surface gravity of the star. While the anomalous light curve of XZ Ceti might be due to the possible location of the star near the red edge, the Fourier parameters $\phi_{21}$ and $\phi_{31}$, which do not seem to depend upon amplitude (Simon and Lee 1981; Simon and Teays 1982), argue against this. In particular it is difficult to understand why none of the stars in Petersen's (1984) sample have Fourier coefficients like those of XZ Ceti even though a number of them are comparable in period and color (Butler, Dickens, and Epps 1978).

Model 2 shows a resonance which could explain XZ Ceti's unusual light curve by analogy with the classical Population I Cepheids (Simon and Teays 1982; Simon and Lee 1981). However, this model has a very low mass, hard to reconcile with the theory of stellar evolution. On the other hand, the anomalous Cepheid model (model 3 ) has too large a mass to interpret it as the product of binary mass exchange. Furthermore, the fundamental mode of model 3 is only very weakly energized.

If we eliminate the F-mode models on the basis of these constraints, we are left with the two overtone representations, models 4 and 5 . Assuming that model 4 is correct, XZ Ceti is a short-period Type II Cepheid (BL Her star) with a distance from the Sun of $0.82 \mathrm{kpc}$ and an elevation from the galactic disk of $0.79 \mathrm{kpc}$. The main difficulty with model 4 is that our (admittedly rudimentary) knowledge of modal selection indicates fundamental (rather than overtone) pulsation at limiting amplitude.

If we accept model 5, then XZ Ceti is an anomalous Population II Cepheid whose high mass results from binary mass 
exchange. In this case its distance and elevation are $1.19 \mathrm{kpc}$ and $1.12 \mathrm{kpc}$ respectively. The absolute blue magnitude $M_{B}$ of $\mathrm{XZ}$ Ceti may be evaluated as follows:

$$
M_{B}=(B-V)_{0}+M_{\text {bol }}-\mathrm{BC},
$$

where $(B-V)_{0}$ and $\mathrm{BC}$ are taken from the interpreted observations, and $M_{\text {bol }}$ is calculated from the model luminosity. The values of $M_{B}$ are given in Table 4. The blue magnitude associated with model 5 is -0.56 , which places XZ Ceti in the middle of the anomalous Cepheid locus in the $M_{B}-\log p$ diagram given by Zinn (1980). No strong objection to the first-overtone anomalous Cepheid model is apparent from any of the data on hand.

Thus we may at present rank the viable models for XZ Ceti as follows: (1) first overtone anomalous Cepheid, and (2) first overtone BL Her star. While the former seems preferable at the moment, further research will be necessary to verify this conclusion. On the observational side, a search for a dim companion to XZ Ceti would be particularly important, while hydrodynamic modeling of a number of the candidates could go part of the way toward clearing up the theoretical uncertainties. It is clear that considerable work needs to be done before the nature of $\mathrm{XZ}$ Ceti can be ascertained with any degree of certainty. If XZ Ceti does indeed turn out to be an anomalous Cepheid, then it would be only the second one known in our Galaxy, and would be by far the brightest, and hence easiest to study, member of this class of stars.

Note added in manuscript.-After completing this manuscript we learned from $J$. Lub that he has determined the reddening of XZ Ceti to be 0.03 . This small reddening would then slightly increase the temperature and gravity results.

The authors wish to thank E. G. Schmidt for helpful discussions of these data. We are also pleased to acknowledge support from the National Science Foundation under grant AST-83 16875.
Aikawa, T., and Simon, N. R. 1983, Ap. J., 273, 346.

Breger, M. 1976, Ap. J. Suppl., 32, 7.

Butler, D., Dickens, R. J., and Epps, E. 1978, Ap. J., 225, 148

Carson, T. R., Stothers, R., and Vemury, S. K. 1981, Ap. J., 244, 230

Cox, A. N. 1980, Space Sci. Rev., 27, 475.

Dean, J. F., Cousins, A. W. J., Bywater, R. A., and Warren, P. R. 1977, Mem R.A.S., 83, 69.

Gutierrez-Moreno, A., Moreno, H., and Cortes, G. 1982, Pub. A.S.P., 94, 722.

Hayes, D. S., and Latham, D. W. 1975, Ap. J., 197, 593.

King, D. S., Cox, J. P., Eilers, D. D., and Davey, W. R. 1973, Ap. J., 182, 859.

Kukarkin, B. V., et al. 1969, General Catalog of Variable Stars (3d ed., Moscow: Sternberg State Astronomical Institute).

Kurucz, R. L. 1979, Ap. J. Suppl., 40, 1

Oke, J. B. 1964, Ap. J., 140, 689.

\section{REFERENCES}

Petersen, J. O. 1984, preprint.

Renzini, A., Mengel, J. G., and Sweigart, A. V. 1977, Astr. Ap., 56, 369.

Schmidt, E. G. 1984, private communication.

Simon, N. R. 1979, Astr. Ap., 75, 140.

Simon, N. R., and Lee, A. S. 1981, Ap. J., 248, 291

Simon, N. R., and Teays, T. J. 1982, Ap.J., 261, 586.

Spangenberg, W. H. 1975, in Proceedings of the Cepheid Modeling Conference and Workshop, D. Fischel and W. M. Sparks (NASA: SP-383), p. 262.

Stellingwerf, R. F. 1975, Ap. J., 195, 414 .

Stothers, R. B. 1983, Ap. J., 274, 20.

Zinn, R. 1980, in Globular Clusters, ed. D. Hanes and B. Madore (Cambridge: Cambridge University Press), p. 191.

Zinn, R., and King, C. R., 1982, Ap. J., 262, 700 